\title{
Early Respiratory Outcomes Following Cardiac Surgery in Patients with COVID-19
}

\author{
khosro barkhordari ${ }^{1}$, mohamad reza khajavi ${ }^{1}$, sepideh nikkhah ${ }^{2}$, Mahmoud Shirzad $^{3}$, \\ sepehr barkhordari ${ }^{4}$, jamshid bagheri ${ }^{1}$, katayun kharazemian ${ }^{3}$, and marjan nosrati ${ }^{3}$ \\ ${ }^{1}$ Tehran University of Medical Sciences \\ ${ }^{2}$ Ministry of Health and Medical Education \\ ${ }^{3}$ Tehran Heart Center \\ ${ }^{4}$ University of California Davis
}

May 11, 2020

\begin{abstract}
Abstract The objective of this study was to describe early respiratory outcomes of asymptomatic COVID-19 patients after cardiac surgery. In this retrospective clinical study (case series) we reviewed and analyzed patient clinical data of 25 covid-19 asymptomatic patients that underwent urgent or emergent cardiac surgery between February 29 and April 10, 2020 in Tehran Heart Center Hospital. Median of age was 63 years (IQR, 52-67), Euro SCORE 7.50 (IQR, 6.5-8.5) and body mass index 26.3 (IQR, 22.5-28.6). 68\% of patients had one or more comorbidities. Hypertension (56\%) was the most common followed by Diabetes type $2(40 \%)$. Off-pump cardiac surgery was done in 4 patients and on-pump on 21 patients with median CPB time of 85 minutes (IQR, 50-147.50). Median anesthesia time was 4.5 hours (IQR, 4-5). Median oxygen index and Fio2 on ventilator were $10 \mathrm{cmH} 20$ (IQR, 9.5-10.5) and 0.64(IQR, 0.60-0.64) respectively. Median pao2/Fio2 was 231(IQR, 184-261). There was one case of extubation failure. The Median intubation time and length of ICU stay were 13 hours (IQR, 9.5-18) and 3 days (IQR, 2-4) respectively. Overall mortality was $16 \%$. Readmission rate to ICU was $16 \%$ with. In this group respiratory outcome was worse with median Pao2/Fio2 84.5 (75-122), oxygen index of 4.38(IQR, 3.77-5.1) and morality rate of 75\%. Conclusion: Based on the results of this study, very early post-cardiac surgery respiratory outcomes in asymptomatic COVID-19 patients are apparently smooth; nonetheless, readmission to the ICU is high. Overall respiratory outcomes are poor especially for those who readmitted to ICU.
\end{abstract}

\section{Early Respiratory Outcomes Following Cardiac Surgery in Patients with COVID-19}

Running title : Respiratory Outcomes PostCardiac Surgery COVID-19

Authors :

Khosro Barkhordari ${ }^{*}$, Mohamad Reza Khajavi ${ }^{2}$, Sepideh Nikkhah ${ }^{3}$ Mahmood Shirzad ${ }^{4}$, Jamshid Bagheri ${ }^{4}$, Sepehr Barkhordari ${ }^{5}$, Katayun Kharazmian ${ }^{3}$, Marjan Nosrati ${ }^{6}$

1- Department of Anesthesiology and Critical Care, Tehran Heart Center, Tehran University of Medical Sciences, Tehran, Iran

2- Department of Anesthesiology and Critical Care, Sina Hospital, Tehran University of Medical Sciences, Tehran, Iran

4-Department of cardiac surgery, Tehran Heart Center, Tehran University of Medical Sciences, Tehran, Iran 3- Food and Drug Bureau, Ministry of Health and Medical Education, Tehran, Iran 
5- College of Biological Sciences, University of California, Davis, USA

6- Department of disease control, Tehran heart center, Tehran, Iran

*Correspondence: kh.barkhordari@gmail.com. Department of anesthesiology and critical care, Tehran heart Center, Tehran University of Medical Sciences, Tehran, IRAN, 1411713138

Tel: +989123903641

Key words : cardiac surgery, respiratory, outcome, COVID-19

Abstract

The objective of this study was to describe early respiratory outcomes of asymptomatic COVID-19 patients after cardiac surgery.

In this retrospective clinical study (case series) we reviewed and analyzed patient clinical data of 25 covid-19 asymptomatic patients that underwent urgent or emergent cardiac surgery between February 29 and April 10, 2020 in Tehran Heart Center Hospital.

Median of age was 63 years (IQR, 52-67), Euro SCORE 7.50 (IQR, 6.5-8.5) and body mass index 26.3 (IQR, 22.5-28.6). $68 \%$ of patients had one or more comorbidities. Hypertension (56\%) was the most common followed by Diabetes type 2 (40\%). Off-pump cardiac surgery was done in 4 patients and on-pump on 21 patients with median CPB time of 85 minutes (IQR, 50-147.50). Median anesthesia time was 4.5 hours (IQR, 4-5). Median oxygen index and Fio2 on ventilator were $10 \mathrm{cmH20}$ (IQR, 9.5-10.5) and 0.64(IQR, 0.60-0.64) respectively. Median pao2/Fio2 was 231(IQR, 184-261). There was one case of extubation failure. The Median intubation time and length of ICU stay were 13 hours (IQR, 9.5-18) and 3 days (IQR, 2-4) respectively. Overall mortality was $16 \%$. Readmission rate to ICU was $16 \%$ with. In this group respiratory outcome was worse with median Pao2/Fio2 84.5 (75-122), oxygen index of 4.38(IQR, 3.77-5.1) and morality rate of $75 \%$.

Conclusion: Based on the results of this study, very early post-cardiac surgery respiratory outcomes in asymptomatic COVID-19 patients are apparently smooth; nonetheless, readmission to the ICU is high. Overall respiratory outcomes are poor especially for those who readmitted to ICU.

\section{Introduction}

Corona virus disease 2019 (COVID -19) has been announced as a pandemic on March 11, by World Health Organization (WHO). Unfortunately, IRAN is one of the hotspot countries among many other countries. Our hospital, Tehran Heart Center, was one of few referral hospitals dedicated to emergency heart surgery patients by Ministry of Health. The lung is the main organ that is affected by this disease. About $80 \%$ of symptomatic COVID-19 patients develop mild disease, and 15\% develop severe disease (with hypoxemia, dyspnea and tachypnea), while $5 \%$ become critically ill with respiratory failure, septic shock and/or multiorgan dysfunction $^{1}$. Abnormalities are visible on chest $\mathrm{CT}$ cans in $85 \%$ of patients ${ }^{2}$. A majority of patients with severe respiratory disease need invasive mechanical ventilation ${ }^{3}$. Inflammatory renal and lung diseases and at extreme acute respiratory distress syndrome (ARDS) and acute renal failure are serious complications of both COVID-19 and cardiopulmonary by-pass (CPB) ${ }^{3,4}$. There are few studies about clinical outcomes of covid-19 patients in intensive care unit. ICU mortality is $22-66 \%$ in nonsurgical patients ${ }^{3,5}$. In a recent large case series in Italy the mortality rate of patients admitted to ICU was about 26 percent $^{3}$. A lot of patients had ARDS and needed high fraction of inspiratory oxygen (Fio2) and positive end expiratory pressure (PEEP). There is a very little information about clinical outcomes of surgical COVID-19 patients. Surgery itself and mechanical ventilation have own impacts on the lung. CPB aggravates these injuries even further in cardiac surgery patients ${ }^{6}$. In a retrospective cohort study of general surgical covid-19 patients, all had lung CT abnormalities after surgery and mortality rate in ICU was $20.5 \%^{7}$. Studies about airway management during surgery in patients with COVID- 19 are rare and we worked based on recommendations and suggestions of experties. ${ }^{8}$ Since at the time of writing of this article, we couldn't find any study regarding post anesthesia respiratory outcomes of cardiac surgery patients. The aim of our study was to analyze 
the early post-anesthesia respiratory outcomes of asymptomatic COVID-19 cardiac surgery patients who underwent urgent or emergent surgery on discern of cardiac surgeon and cardiologist. Experts recommend lung protection strategy for intubated COVID-19 patients, like other ARDS patients ${ }^{9,10}$. We worked based on that recommendations. Since at the time of writing this article there was not any study in cardiac surgery patients. We think our small data can provide knowledge about post-operation respiratory course in cardiac surgery patients.

2. Materials and Methods:

Patients:

In this retrospective study data of patients with COVID-19 who underwent urgent or emergent cardiac surgery in Tehran Heart Center Hospital between February 29 and April 10, 2020 and fulfilled our study criteria were reviewed and analyzed. Informed consent obtained from all patients regarding using their medical information for research purposes. This study was approved by research committee of Tehran heart center for medical record review. The diagnosis of COVID-18 was the responsibility of a team comprising a radiologist, and infectious disease specialist, and Intensivist based on positive reverse transcription-polymerase chain reaction(RT-PCR) tests or chest $\mathrm{CT}$ according to the WHO interim guidance ${ }^{10}$. Patients were asymptomatic at admission time. All of the patients were receiving hydroxychloroquine and azithromycin and some of them antiviral drugs. General anesthesia induced with routine anesthetic drugs by in-charged anesthesiologists. Majority of patients received Midazolam, Fentanyl, Propofol, and Atracurium as induction drugs. For maintenance propofol and fentanyl infusions and divided doses of Atracurium and Panccuronium were used as needed. Eight of the patients received Isoflurane, in addition to above drugs, both during induction and maintenance of anesthesia. On-pump cardiac surgery was done in 21 patients and 4 had off-pump surgery. In open heart ICU, patients were put on routine post cardiac surgery care and monitoring. Ventilator parameters were adjusted by anesthesiologists and intensivists in charge of patients based on hemodynamic, respiratory and ABG indices. Based on hemodynamic and respiratory indices low tidal volumes $(6-8 \mathrm{ml} / \mathrm{kg}$ IBW) and different amounts of PEEP were used. Depend on clinical conditions of patients, our ICU early extubation protocol strategy followed as could as possible. Fluid management was based on hemodynamic monitoring indices, urine output, and clinical judgments and also conservative volume strategy was applied as could as possible.

Data collection:

The patient characteristics and data collected from medical records and ICU flow sheets. Data of patients with age[?] 18, active respiratory disease, patients with renal and hepatic failure, history of uncontrolled respiratory diseases excluded from reviewing. Data in ICU included hemodynamic parameters, Mean Airway Pressure (MAWP), amount of PEEP, Fio2, Pao2, Paco2 ,fluid balance in three day, serum creatinine, postoperative bleeding, reopening of chest due to surgical complications, amount of received PRBC and blood components among others. Euro SCORE II was used for risk stratification ${ }^{11}$.

\section{Outcomes:}

Our early respiratory outcomes were extubation failure rate, intubation time, Pao2/Fio2 ratio, Oxygen index, and Mean Airway Pressure (MAWP). Other outcomes were: length of stay in ICU (ICULOS), readmission to ICU, AKI, and mortality in 30 days after surgery. Pao2/Fio2 ratio and Oxygenation index calculated from computation of pao2, Fio2, MAWP variables based on their specific formula. Based on Berlin definition, severity of ARDS in patients was determined using Pao2/Fio2 ratios $^{12}$. AKI of patients defined based on changes on creatinine according to KDIGO guidelines for postoperative patients ${ }^{13}$.

Statistical analysis:

Continuous variables are presented as mean and median. Categorical variables were expressed as frequencies and percentages. Statistical analysis was performed by SPSS software version 21.

3. Results: 
A total 25 patients who had COVID-19 and underwent cardiac surgery were enrolled in the study. 21 patients had positive test and 4 had positive chest HRCT. Basic characteristics of patients are shown in Table 1. Seventy two percent of patients were male. The median of age was 63 years (IQR, 52-67). Median EuroSCORE and body mass index were 7.50 (IQR, 6.5-8.5), 26.3 (IQR, 22.5-28.6), respectively. 68\% of patients had one or more comorbidities. Hypertension (56\%) was the most common followed by Diabetes type $2(40 \%) .20 \%$ of patients were Cigarette smokers. Mean left ventricular ejection fraction (LVEF) was 43.53 $\%(\mathrm{SD} \pm 8.40)$. Four patients $(16 \%)$ had history of respiratory diseases, one of them asthma and three COPD, but their diseases were under controlled. Off-pump cardiac surgery was done in 4 of our patients and $21(84 \%)$ patients underwent on-pump with median CPB time of 85 minutes (IQR, 50-147). The types of surgery were diverse that is shown in Table 2. Median anesthesia time was 4.5 hours (IQR, 4.5-5). 14 patients received inotropes during surgery with maximum doses of norepinephrine $0.08 \mu / \mathrm{kg} / \mathrm{min}$, dobutamine $10 \mu / \mathrm{kg} / \mathrm{min}$ and epinephrine $0.05 \mu \mathrm{g} / \mathrm{kg} / \mathrm{min}$. One patient needed IABP. Mean Pao2, Fio2, Pao2/Fio2 ratio, MAWP and Oxygen index during first 6 hours after surgery were $146(\mathrm{SD} \pm 27.99) \mathrm{mmHg}, 0.63(\mathrm{SD} \pm 0.48), 232.82$ $(\mathrm{SD} \pm$ 49.23), $10 \mathrm{cmH} 20(\mathrm{SD} \pm 1.20)$ and 4.47( $\mathrm{SD} \pm 1.13)$, respectively. Median PEEP was 5.5(5.25-6). Base on Berlin definition of ARDS, at entering to ICU one patients had severe ARDS, 12(44.4\%) moderate and $10(37 \%)$ mild ARDS but after 8 hours there was no severe ARDS, 6 (24\%) had moderate and 17(48\%) mild ARDS. $40 \%$ of patients had AKI in post-operative day 3. 3(12\%) patients had reoperation due to bleeding. PRBC transfusion done in $14(56 \%)$ of patients. Mean hemodynamic parameters until extubation are showed on Table 1. Majority of patients had normal sinus rhythm (84\%), $18 \%$ sinus tachycardia, $12 \%$ atrial fibrillation and 1 persistent ventricular tachycardia that needs DC Shock. One of patients re-intubated after 8 hours of extubation. Median intubation time was 13hours (IQR, 18-9.5) and median first ICU LOS was 3 days (2-4). These figures are not include the patients readmitted to the ICU. Four patients $(16 \%)$ readmitted to ICU with median time from discharge to readmission to ICU of 4.5 days (IQR, 3-6.5). In the readmitted patients the Fio2 and Oxygen index, and PEEP were higher, and Pao2/Fio2 ratio was lower than main group. Table 3 .Three $(75 \%)$ of these 4 patients died.

\section{Conclusions:}

To the best of our knowledge, at the time of the writing of the present study, the literature contains no investigation on the respiratory outcomes of post-cardiac surgery patients with COVID-19. In this case series, we retrospectively reviewed basic characteristics and clinical data of 25 patients that underwent emergent or urgent cardiac surgeries at the discretion of cardiac surgeons and cardiologists. As our country is one of the several global hot spots of COVID-19, all our cardiac surgery patients routinely preoperative COVID-19 test and computed tomography (CT). The diagnosis of COVID-18 was the responsibility of a team comprising a radiologist, and infectious disease specialist, and intensivist based on positive reverse transcription-polymerase chain reaction(RT-PCR) tests or chest CT according to the WHO interim guidance ${ }^{10}$. Twenty-one patients tested positive for COVID-19, and 4 were diagnosed based on their chest CT and history of close contact with COVID-19 patients. A recent retrospective study in China described clinical outcomes of general surgical patients with diverse types of surgeries ${ }^{7}$. In comparison with the patients in that study our patients were older. (mean age, 63 years). The median EuroSCORE of our patients was 7.50 (IQR, 6.5-8.5) indicating higher risk of morbidity and mortality. $68 \%$ of our patients had at least one medical condition this is more than the surgical patients that needed ICU admission in the Chinese study. With respect to medical conditions, our patients were similar to the nonsurgical patients admitted to the ICU in a study by Grasseli et al. ${ }^{3,7} \mathrm{Chiming}$ in with other studies, hypertension was the most common comorbidity in our investigation. Hemodynamic complications and both cardiogenic and septic shocks are not uncommon in COVID-197,14.But our patients had relatively acceptable hemodynamic parameters after surgery.Fourteen (64\%) patients received usual doses and four $(16 \%)$ needed high doses of inotropes in current study. In a study by lei et al. on non-cardiac surgery patients, $53 \%$ of the patients admitted to the ICU had shock and $33 \%$ had cardiac arrhythmias. Because inotropes are frequently used to support hemodynamics in cardiac surgery patients, we cannot compare the incidence rate of shock between that study and ours; nonetheless, overall, our patients were hemodynamically stable after surgery. One of our valve surgery patients needed IABP in operating room. Another patient that underwent on-pump CABG needed DC shock twice, which was because of sustained 
episodes of ventricular fibrillation. This patient had no history of any medical conditions and subsequently had a straightforward postoperative ICU course. In a retrospective study that compared postoperative outcomes between patients undergoing emergency CABG and those undergoing elective CABG, morbidity and mortality were higher in former group ${ }^{15}$. In that study the mean CPB time was $80.2 \pm 39.7$ minutes, which is shorter than our CPB time $(99.75 \pm 63.5)$. Nevertheless, given the diversity in our surgical operations, this $\mathrm{CPB}$ time is expectable. In our study rate of acute kidney injury (AKI) on the third postoperative day was $40 \%$ which is higher than the rate in COVID-19 ICU patients following general surgery and non COVID emergency cardiac surgery patients. ${ }^{7,15}$ Although some anesthetic agents may poses anti-inflammatory effects, surgery itself, specially cardiac surgery, and CPB cause inflammatory response and acute lung injury 16,17. Upon admission to ICU, based on the Berlin ARDS definition, only one of our patients had severe ARDS, while the majority of patients had moderate (44.4\%), or mild ARDS (37\%). The mean airway pressure (9.96 $\mathrm{SD} \pm 1.48$ ) was not unexpectedly high, indicating that fluid overload and heart stunning might also have a role in hypoxemia. Moreover, lesser severity of ARDS may be explained by the fact that our patients were asymptomatic and anesthetics and muscular relaxants affect the compliance and resistance of the lung. The median airway pressure and Fio2 of intubated patients were $10 \mathrm{cmH} 20$ (IQR, 9.50-10.50), and 0.64 (IQR, 0.60-0.64) respectively, which are lower than the values reported in the study by Grasselli et $\mathrm{al}^{3}$. In addition the median of Pao2/Fio2 was 231(IQR, 184-261), which was higher than nonsurgical intubated patients. Our patients were asymptomatic, whereas the ICU patients in the study by Grasselli and colleages srffered from severe pneumonia. We used the amount of PEEP based on hemodynamic and respiratory parameters. Because at least for the first hours of postoperative period, the majority of cardiac surgery patients have hemodynamic instability, the median PEEP in our patients [6 IQR, (5-6)] was lower than in nonsurgical ventilated patients. Oxygenation was acceptable with this amount of PEEP and Fio2, and the median oxygen index was [4.3 (IQR, 3.8-5.2)] and relatively constant over time. Emergent or urgency surgery, old age, comorbidities, and high frailty scores are associated with longer mechanical ventilation in cardiac surgery patients ${ }^{18}$. Our mean intubation time was 22.25 hours and all the patients extubated within 99 hours post procedurally, and $44 \%$ of them were extubated within twelve hours after admission to the ICU. It is worth noting that this figure does not include the patients that readmitted to the the ICU. We had one case of extubation failure, this patient was re-intubated because of hypoxemia and respiratory failure 8 hours later. He was again extubated after 24 hours and discharged from the ICU; however, 2 days later, he was readmitted to ICU with severe respiratory insufficiency, septic shock, ventricular arrhythmia and unfortunately expired 5 days afterward. One of our patients died in the first 7 days of ICU stay. This patient had fever, arrhythmias from sinus tachycardia to sustained ventricular fibrillation and agitation died at $7^{\text {th }}$ day. He was never weaned from ventilator, with severe ARDS, metabolic acidosis, bradycardia and asystole representing the clinical pictures of his death. Unlike the post- surgery patients in the study by Lei et al, in which fever was most common symptom, a low spo2 $(<87 \%)$ was the most common first sign in post-ICU and wards (80\%). Fever (72\%), respiratory distress $(64 \%)$, headache $(28 \%)$, and cough $(28 \%)$ constituted the common signs and symptoms. Majority of patients improved with routine supportive cares. Four patients were readmitted after discharge from the ICU due to respiratory and hemodynamic problems.

Respiratory and ventilator indices during this second stay in the ICU were unfavorable. (Table 3). Based on the Berlin definition, 3 (75\%) of patients suffered from severe ARDS and 1 had moderate ARDS. Median PEEP and Fio2 was much higher than main group. In this group Lymphocyte count was lower and C-reactive protein (CRP) was higher than in the main group of patients. CRP in cardiac surgery patients is already high due to inflammation; still, in our re-admitted group, the CRP level was much higher than in the main group. Three $(75 \%)$ of these patients died, which by comparison with the nonsurgical ICU patients in the study by Grasselli et al is high. Acute viral myocarditis was the first differential diagnosis of 2 of these patients as decided by the cardiologist in charge. These patients had severe tachycardia, low EF, and high values of troponin enzymes (Table 3). This indicates that symptomatic post-cardiac surgery patients carry a very high risk of mortality, although the early postoperative respiratory and hemodynamic outcomes of our patients appeared acceptable with only 1 case of extubation failure and mortality in the first week of post-operative period. High rate of ICU readmission (16\%) and the very high mortality rate (75\%) among this group were alarming, however. 
In light of the results of the present study, it can be concluded that the very early postoperative respiratory outcomes in asymptomatic COVID-19 emergent and urgent cardiac surgery patients are smooth; nonetheless, readmission to the ICU is high. Thus in this group of patients we should expect very high rates of severe ARDS and mortality. We suggest that cardiac surgical operations be postponed unless they are emergent.

This study has several limitations. First, this study is a retrospective study and data collected from medical records and flow sheets so some data are missed. Secondly, the sample size is too small to compare between groups. Third, patients in post ICU and other wards were not monitored same as open heart ICU.

\section{References}

1. Wu Z, McGoogan JM. Characteristics of and important lessons from the coronavirus disease 2019 (COVID19) outbreak in China: summary of a report of 72314 cases from the Chinese Center for Disease Control and Prevention. Jama. 2020. 2. Guan W-j, Ni Z-y, Hu Y, et al. Clinical characteristics of coronavirus disease 2019 in China. New England Journal of Medicine. 2020. 3. Grasselli G, Zangrillo A, Zanella A, et al. Baseline characteristics and outcomes of 1591 patients infected with SARS-CoV-2 admitted to ICUs of the Lombardy region, Italy. Jama. 2020. 4. Naveed A, Azam H, Murtaza HG, Ahmad RA, Baig MAR. Incidence and risk factors of Pulmonary Complications after Cardiopulmonary bypass.Pakistan journal of medical sciences. 2017;33(4):993. 5. Murthy S, Gomersall CD, Fowler RA. Care for critically ill patients with COVID-19. Jama. 2020. 6. Xu S, Liu J, Li L, et al. Cardiopulmonary bypass time is an independent risk factor for acute kidney injury in emergent thoracic aortic surgery: a retrospective cohort study. Journal of cardiothoracic surgery. 2019;14(1):90. 7. Lei S, Jiang F, Su W, et al. Clinical characteristics and outcomes of patients undergoing surgeries during the incubation period of COVID-19 infection. EClinicalMedicine. 2020:100331. 8. Namendys-Silva SA. Respiratory support for patients with COVID-19 infection. The Lancet Respiratory Medicine. 2020;8(4):e18. 9. Alhazzani W, Møller MH, Arabi YM, et al. Surviving Sepsis Campaign: guidelines on the management of critically ill adults with Coronavirus Disease 2019 (COVID-19). Intensive care medicine. 2020:1-34. 10. World Health Organization. Clinical management of severe acute respiratory infection when novel coronavirus (2019-nCoV) infection is suspected: interim guidance, 28 January 2020. Organization;2020. 11. Ad N, Holmes SD, Patel J, Pritchard G, Shuman DJ, Halpin L. Comparison of EuroSCORE II, original EuroSCORE, and the Society of Thoracic Surgeons risk score in cardiac surgery patients. The Annals of thoracic surgery. 2016;102(2):573-579. 12. Force ADT, Ranieri V, Rubenfeld G, Thompson B, Ferguson N, Caldwell E. Acute respiratory distress syndrome. Jama.2012;307(23):2526-2533. 13. Kellum JA, Lameire N, Group KAGW. Diagnosis, evaluation, and management of acute kidney injury: a KDIGO summary (Part 1). Critical care. 2013;17(1):204. 14. Fried JA, Ramasubbu K, Bhatt R, et al. The Variety of Cardiovascular Presentations of COVID-19. Circulation. 2020. 15. Schumer EM, Chaney JH, Trivedi JR, Linsky PL, Williams ML, Slaughter MS. Emergency coronary artery bypass grafting: indications and outcomes from 2003 through 2013. Texas Heart Institute Journal.2016;43(3):214-219. 16. Cruz FF, Rocco PRM, Pelosi P. Anti-inflammatory properties of anesthetic agents. Critical Care. 2017;21(1):67. 17. Manrique AM, Vargas DP, Palmer D, Kelly K, Litchenstein SE. The effects of cardiopulmonary bypass following pediatric cardiac surgery. Critical Care of Children with Heart Disease: Springer; 2020:113-129. 18. Hessels L, Coulson TG, Seevanayagam S, et al. Development and validation of a score to identify cardiac surgery patients at high risk of prolonged mechanical ventilation. Journal of cardiothoracic and vascular anesthesia. 2019;33(10):2709-2716.

\section{Hosted file}

table.1.docx available at https://authorea.com/users/320064/articles/449675-earlyrespiratory-outcomes-following-cardiac-surgery-in-patients-with-covid-19

\section{Hosted file}

Table2 . docx available at https://authorea.com/users/320064/articles/449675-early-respiratoryoutcomes-following-cardiac-surgery-in-patients-with-covid-19

\section{Hosted file}


table. 3.docx available at https://authorea.com/users/320064/articles/449675-earlyrespiratory-outcomes-following-cardiac-surgery-in-patients-with-covid-19 